\title{
Manual de emergencias aéreas y terrestres para servicio aéreo a territorios nacionales SATENA
}

\section{Los manuales y procedimientos para la atención de emergencias se convierten en herramienta clave para reducir los riesgos y las posibilidades de que un incidente en vuelo o en tierra de una aeronave se convierta en un siniestro trágico.}

\section{MY. Julián Fernando Gómez Lince MY. Juan Guillermo Támara Melo}

La seguridad aérea es un tema de preocupación mundial, recientemente en la Unión Europea (UE), se ha aprobado una drástica legislación (Reglamento (CE) № 21111/2005 de 14 de diciembre de 2005) para el establecimiento de normas comunes para mantener el espacio aéreo europeo libre de compañías aéreas y aeronaves que se considere no son seguras.

El 22 de marzo de 2006 se publicó una primera lista comunitaria de compañias aéreas prohibidas en la Unión Europea. Puesto que la Comisión tiene la obligación de garantizar que la lista esté actualizada y la de verificar, como mínimo cada tres meses, si necesita una actualización, posteriormente se publicó una revista revisada el 20 de junio de 2006 '. La actualización de la lista requiere una permanente vigilancia de todas las partes implicadas, es decir, la Comisión, la European Aviation Safety Agency (Agencia Europea de Seguridad Aérea) y los Es- tados miembros. Éstos tienen la obligación de ejecutar las prohibiciones de explotación en su territorio. Además, los Estados miembros y la Agencia Europea de Seguridad Aérea están obligados a comunicar a la Comisión toda la información que pueda ser relevante para la actualización de la lista. Los criterios para la misma son transparentes y están estrechamente vinculados a la seguridad².

Atendiendo estos estándares internacionales, la aviación colombiana, y especialmente SATENA, tienen la obligación de adoptar, mantener y renovar sus manuales y procedimientos para la atención de emergencias aéreas o terrestres a fin de alcanzar las calificaciones de la aviación internacional en esta materia.

El manual propuesto en este trabajo indudablemente se enmarca dentro de esos objetivos de la aviación internacional; si bien muchas de las apreciaciones de agencias como IATA pueden no tener una aplicación en los vuelos

'NOTICIAS DE LA UE. La UE en España. Derechos de los pasajeros \& seguridad aérea, Prioridad de la Comisión. Bruselas, 18 de julio de 2006, Disponible en: http://ec.europa. eu/spain/novedades/18_07_2006_2_es.htm 
nacionales de SATENA, pero no por ello deben excluirse de la reglamentación ordinaria puesto que en cualquier momento, por la naturaleza de la empresa, podría atender demandas de vuelos internacionales en casos concretos como por ejemplo las operaciones de paz. Indudablemente el manual de atención de emergencias aéreas y terrestres de SATENA debe enmarcarse también en un Sistema Integrado de Gestión que permita atender los requerimientos de las normas ISO 9001:20003, consultar los lineamientos de los Programas de Garantía de Calidad ATS de la OACl, aprobado a través de la Conclusión 10/18 del GREPECAS $/ 10^{4}$ y alcanzar los índices de calidad internacional de la industria aeronáutica.

\section{Descripción}

En la actualidad toda empresa, en especial las relacionadas con la industria aeroespacial, deben tener un Plan de Emergencias ${ }^{5}$. En este momento SATENA no cuenta con un manual de emergencias que integre los procedimientos a seguir en caso de accidentes o incidentes aéreos, terrestre $s^{6} y$ actos de interferencia ilícita. Dado que la empresa adelanta el proceso de certificación ante la Unidad Administrativa Especial de Aeronáutica Civil Colombiana; es indispensable contar con un manual de este tipo tanto para la empresa como para cada una de las agencias donde los aviones de SATENA cumplen sus itinerarios. En otras palabras, SATENA requiere de un $\mathrm{PEAT}^{7}$, es decir que necesita de un Manual de consulta interna que sirva como guia para todas aquellas personas que trabajan en SATENA y adquieren funciones asi como responsabilidades directas cuando se presenta una situación de emergencia que comprometa la seguridad de una de las aeronaves de la Empresa.

El Manual de Emergencias Aéreas y Terrestres es una herramienta indispensable para SATENA ya que le permitirá estandarizar los procedimientos a seguir por parte de las directivas y personal de rescate de la empresa en caso de algún siniestro, desastre o accidente de una de sus aeronaves o en las instalaciones de la misma. Un manual con estas caracteristicas permitirá que la empresa sea una aerolínea competitiva y certificada por la Unidad Administrativa Especial de Aeronáutica Civil (UAEAC). La Organización de Aviación Civil Internacional (OACI), periódicamente controla a sus afiliados con el fin de exigir el cumplimiento de normas y procedimientos de seguridad aérea en general, tanto de las empresas nacionales como de las extranjeras.

A continuación se mencionan los objetivos de la investigación, la metodología empleada y el resultado obtenido.

\section{Objetivos}

\section{General}

Elaborar un manual de emergencias aéreas y terrestres para SATENA que permita estandarizar los procedimientos a seguir en caso de accidentes o incidentes aéreos, terrestres $y$ actos de interferencia ilícita.

\section{Especificos}

- Recopilar la información existente en documentos de empresas dedicadas a la aviación, a nivel nacional e internacional, en el área de emergencias aéreas y terrestres.

- Determinar la percepción de los pilotos y personal administrativo de SATENA acerca de la situación actual en el área de seguridad en lo que respecta a emergencias aéreas y terrestres a través de la aplicación de una encuesta.

- Seleccionar los contenidos temáticos que serán incluidos en el manual de emergencias aéreas y terrestres de SATENA a partir de la información recopilada.

\section{Metodología}

Este trabajo de investigación es de tipo descriptivo y documental, porque permite describir los procedimientos y acciones a seguir en caso de emergencia, con aeronaves e instalaciones de la empresa. Además, se recopila

\footnotetext{
3El certificado 150 9001:2000 de la Organización de Seguridad Aérea de la Autoridad Federal de Aviación de Estados Unidos, es un magnifico ejemplo del valor que la norma internacional de gestión de la calidad aporta a un organismo público de gran envergadura y complejidad, cuya misión es delicada y esencial.

"NACC/DCA/2-NI/35. Organización de Aviación Civil Internacional 26/09/05

se entiende por el mismo a un conjunto de medidas adoptadas por la empresa para coordinar acciones de atención y recuperación frente a una situación de crisis o evento desastroso.

- Accidentes o incidentes terrestres hace referencia a siniestros o desastres ocurridos en las instalaciones de la empresa.

? Plan de Emergencias Aéreas y Terrestres, es un documento escrito, su aplicación tendrá lugar en muy pocas ocasiones. Por tal razón, es importante el entrenamiento del PEAT ya que éste permite detectar las debilidades y probar las capacidades de la empresa para responder a una eventual situación de este tipo. Se sugiere su práctica con regularidad y aunque afgunos ejercicios pueden ser desarrollados desde el escritorio, otros requieren el entrenamiento en el campo, siendo ambos muy importantes.
} 
y analiza información relevante para lograr determinar cuáles son los procedimientos a seguir durante una emergencia aérea o terrestre para obtener resultados rápidos y efectivos en lo que respecta a bús-

queda, rescate, asistencia, salvamento y evacuación de las personas y material involucrado.

\section{Resultados}

El Manual se divide en dos secciones, la primera consta de ocho capítulos y corresponde a la atención de las emergencias aéreas. La segunda sección tiene diez capitulos y hace alusión a la atención de las emergencias en tierra.

La primera sección presenta en el primer capítulo un glosario de términos de aviación. En el segundo se plantean las responsabilidades y funciones del Estado, de SATENA, asi como las de la UAEAC y la Fuerza Aérea Colombiana. En el capitulo tercero se brinda información detallada acerca de que es un accidente e incidente aéreo y cómo se debe actuar frente al mismo. En el capitulo cuarto se describe qué es una interferencia ilicita y qué debe hacer la empresa frente a este evento. En el capitulo quinto se hace referencia al manejo de las mercancías peligrosas. En el capítulo sexto se indica qué debe hacer la empresa en caso de que una aeronave se encuentre retrasada o extraviada. En el capítulo séptimo se da información expresa acerca de cómo efectuar las comunicaciones, en caso de presentarse una emergencia aérea, con la prensa y los familiares, entre otros. En el capítulo octavo se especifica cómo deben realizarse los simulacros de emergencia, asi como los posibles tipos de simulacro.

La segunda sección presenta en el primer capítulo las generalidades de lo que implica un plan de emergencia. En el capítulo segundo se da información general sobre la ubicación e instalaciones de la empresa. En el capítulo tercero se establece cómo identificar las amenazas y se propone una metodología de trabajo para este fin. En el capítulo cuarto se da información sobre cómo realizar un análisis de vulnerabilidad en las personas, los recursos, los sistemas y procesos y se establecen las medidas de intervención para disminuir el nivel de riesgo. En el capitulo quinto se presenta un inventario de recursos internos y externos para la atención de emergencias. En el capítulo sexto se muestran las acciones a realizar a través de un plan de acciones formativas. En el capitulo séptimo se brinda el Plan Operativo para el control de emergencias con sus respectivas actividades. En el capítulo octavo se estructura la organización para emergencias, dejando claramente establecidas las funciones del comité coordinador de emergencia y las brigadas de emergencia. En el capítulo noveno se presenta el Plan de evacuación y los procedimientos que se deben seguir para ello. Finalmente, en el capitulo décimo se muestran los procedimientos a seguir en caso de incendio, de atentado terrorista, de toma de las instalaciones, para la atención médica de emergencia, de derrame de liquido y de fuga de gas.

\section{Conclusiones}

Un manual para la atención de emergencias áreas y terrestres es una herramienta importante porque reduce los riesgos y las posibilidades de que un incidente en vuelo o en tierra de una aeronave se convierta en un siniestro trágico; dado que establece normas claras y procedimientos precisos para cuando se pueda presentar una situación como las que en él se describen.

Finalmente, la elaboración de este manual muestra de manera especifica y pormenorizada cuáles son los procedimientos y responsabilidades que se deben llevar a cabo, asi como las acciones iniciales, para asegurar un rápido y sistemático esfuerzo de búsqueda, rescate, asistencia, salvamento y evacuación.

\section{Bibliografia}

Gómez, J\& Támara, J. Manual de Emergencias Aéreas y Terrestres para

Servicio Aéreo a territorios nacionales (Satena). Trabajo de grado. IMA, Bogota, 2007. 